\title{
Noise Reduction in HIPERLAN/2 System Integrated with Alamouti Technique
}

\author{
Maan A. Yahya \\ $\{$ dr.maan@ntu.edu.iq \} \\ Ninevah Technical Institute, Northern Technical University, Mosul, Iraq
}

\begin{abstract}
HIPERLAN/2 system integrated with Alamouti technique is presented, where multiple input multiple output system including space time diversity have been employed into HIPERLAN/2 system. This method, noise reduction is applied when the transmitted antennas are closely located to the receive antennas, and they are working in a full-mode duplexing. Several factors for testing the performance of the modified system such as changing the modulation scheme and the feedback accuracy have been considered, the system performance was tested in terms of bit error rate (BER) and the signal to noise ratio (SNR). MATLAB and Simulink are used for simulating and evaluating the suggested system. the performance of the suggested system is mainly depending on the feedback mismatch amount. The modified system performance enhances as the feedback accuracy increases.
\end{abstract}

Keywords: open-loop transmit diversity, HIPERLAN/2, noise cancellation, space-time diversity, signal to noise ratio, bit error rate.

\section{Introduction}

Increasing the data rate and utilizing the available bandwidth effectively can be achieved by employing multiple input multiple output (MIMO) system [1-7]. Wireless communications systems can be divided into four categories according to the antennas number at the transmit and the receive sides. The first category, uses one antenna for transmitting and another one for receiving, is called Single Input Single Output (SISO). Whereas the second one, has an antenna for transmitting and multiple receiving antennas, is called Single Input Multiple Output (SIMO). The third type is called Multiple Input Single Output (MISO), where multiple antennas are used for transmitting and one antenna for receiving. The last type is the multiple input multiple output 
(MIMO), where multiple antennas are used on both sides. The first attempt of transmitting radio signal wirelessly was done by Guglielmo Marconi in 1896. After that, a lot of research have been carried out by scientists to reduce the impairments effect caused by the environment [8]. Simultaneously, reducing the effect of multipath caused by the obstacles was the main target of researchers in wireless communication systems for spectral efficiency enhancement. Jack Winter, at Bell laboratories, developed the first MIMO system; where it was a start point for using more than one antenna in wireless communications [9-13]. Later in 1993, T. Kailath and A. Paulraj proposed the spatial diversity principle [14]. The main advantages of MIMO systems are spatial multiplexing, interference reduction, diversity gain, and array gain. Nevertheless, both maximum diversity and maximum multiplexing cannot be implemented concurrently, so a trade-off between them should be taken $[9,15]$.

Space-time coding was suggested in last century, where the receiver knows the code applied by the transmitter. Nevertheless, the Alamouti code is the most important schemes where it breaks this rule, where no feedback is required to improve the system performances.

In addition, the IEEE 802.11 standards alternative is the HIgh PERformance wireless Local Area Network (HIPERLAN). it can support up to $54 \mathrm{Mbit} / \mathrm{s}$ and works in $5 \mathrm{GHz}$ frequency band, in addition, it has four subclasses which are HIPERLAN/1 (the first version), HIPERLAN/2 (the second version), HIPERACCESS and HIPERLINK, [16]. In this research paper, an overview regarding Alamouti technique will be given, after that, an HIPERLAN/2 system summary will be disscused, then the integration of the HIPERLAN/2with the Alamouti technique will be discussed. Finally, the modified system results will be discussed and the conclusions will be drawn.

\section{Overview of Alamouti Technique}

The simplest technique used in the transmit diversity is the Alamouti technique, where no feedback is required from the receiver; nevertheless, it introduce additional computation complexity in the transmitting side. The data rate, capacity, signal quality and error performance are the main factors improved by this technique improves at the receiver side and it does not require bandwidth expansion. Also, in wireless systems, the coverage area can be increased by using The Alamouti scheme. Likewise, The Alamouti effect on capacity can be clearly seen in systems limited by the multipath phenomenon. Two cases were studied by Alamouti; the first case, the system is assumed to have two antennas for transmitting and one antenna for the receive side, while in the second case, the system assumed to have two antennas at the transmitting side and another two in the received side. This suggested system generates symbols depending on Mary modulation, where each symbol can be represented by a complex value. Alamouti strategy uses two-time slots in sending the symbols. The first symbol $s_{1}$ is sent by the first transmitter while the second symbol $s_{2}$ is sent by the second transmitter during the first time slot. Whereas at the second time slot, the $-s_{2}^{*}$, is sent by the first transmitter and $s_{1}^{*}$ symbol is sent by the second transmitter as illustrated in the next equation $[1,3,7]$ : 


$$
S=\left(\begin{array}{cc}
s_{1} & -s_{2}^{*} \\
S_{2} & s_{1}^{*}
\end{array}\right)
$$

where the columns represent the time slots for each transmitter, and the rows represents the transmitting antennas. The Alamouti key feature orthogonality between symbols in both time slots (i.e., $s_{1} s_{2}^{*}-s_{2}^{*} s_{1}=0$ ), in addition, the channel between the transmit and the receive side is assumed to be constant for the period of two time slot. Moreover, the receiver should have a knowledge of the channel. Where the total number of channels depends on the number of transmit and receive antennas. Therefore, two channels will be between two transmitting antennas and one receiving antenna:

$$
H=\left[h_{1} h_{2}\right]
$$

where: $\mathrm{h} 1$ and $\mathrm{h} 2$ are the characteristics of channels between the first user transmitting antennas and the second user receiving antenna. The channels can be expressed as a complex value:

$$
\begin{aligned}
& h_{1}=\alpha_{1} e^{j \emptyset_{1}} \\
& h_{2}=\alpha_{2} e^{j \emptyset_{2}}
\end{aligned}
$$

By using Equation (1), and for two time slots, the received signal will be:

$$
\begin{gathered}
y_{1}=h_{1} s_{1}+h_{2} s_{2}+n_{1} \\
y_{2}=-h_{1} s_{2}^{*}+h_{2} s_{1}^{*}+n_{2}
\end{gathered}
$$

where $y_{1}$ and $y_{2}$ are the received signal for the two slots respectively. Then, the two-time slots will be combined and sent to the maximum likelihood detector. Where the combined signals can be represented as:

$$
\begin{aligned}
& \hat{s}_{1}=y_{1} h_{1}^{*}+y_{2}^{*} h_{2} \\
& \hat{s}_{2}=y_{2} h_{1}^{*}-y_{1}^{*} h_{2}
\end{aligned}
$$

The equations of Maximal Ratio Combining (MRC) are different from the combined signals in Equations (7) and (8) as shown below:

$$
\begin{gathered}
\hat{s}_{1}=\left(\alpha_{1}^{2}+\alpha_{2}^{2}\right) s_{1}+h_{1}^{*} n_{1}+h_{2} n_{2}^{*} \hat{s}_{1}=y_{1} h_{1}^{*}+y_{2}^{*} h_{2} \\
\hat{s}_{2}=\left(\alpha_{1}^{2}+\alpha_{2}^{2}\right) s_{2}-h_{1} n_{2}^{*}+h_{2}^{*} n_{1}
\end{gathered}
$$

It is clear that the equations (9) and (10) are equivalent to MRC equations. Nevertheless, the noise components phase is the only difference and do not affect the SNR.

Furthermore, many applications require higher diversity where increasing the diversity can be achieved by increasing the antennas number in both transmit and receive sides. 


\section{HIPERLAN/2 System}

European Telecommunications Standards Institute (ETSI) developed the HIPERLAN standard, which is a wireless communication standard that responsible for providing a digital high-speed data rate [13, 17-19]. HIPERLAN standard works in the $5 \mathrm{GHz}$ band and relay on Orthogonal frequency-division multiplexing (OFDM) [20]. |The system diagram is shown in Fig. 1.

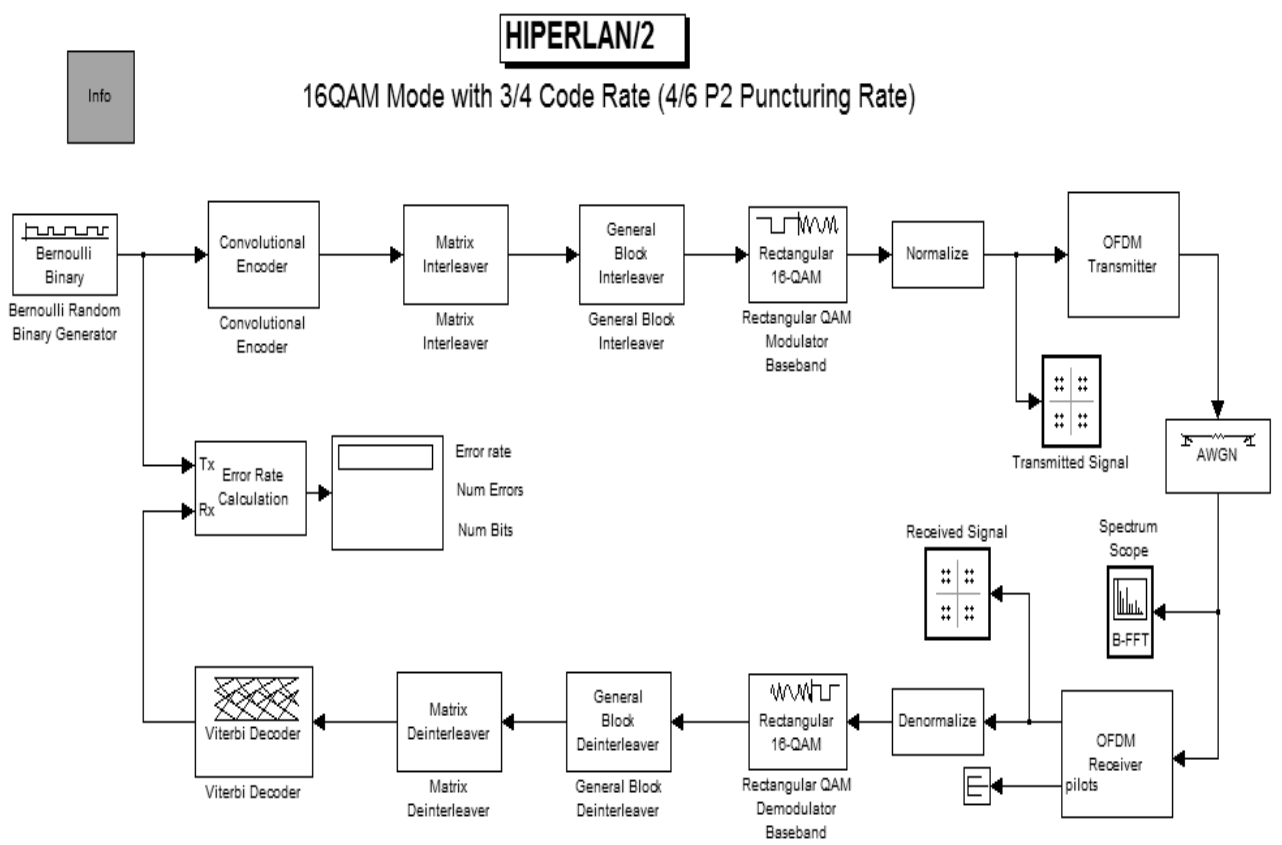

Fig. 2. Classical HIPERLAN system.

In MATLAB software, the HIPERLAN/2 system consists of several block diagrams; where a binary data which consists of ones and zeros that has certain probability of zero distribution is generated by the Bernoulli random generator, after that the convolutional coder that codes the generated data using a certain expression of polynomial. In addition, the convolutional encoder has the ability to puncture the encoded data where not the whole data will be sent. It should be noticed that the punctured data index should be identified by both the transmitter and the receiver. After that, the data will be interleaved firstly using matrix interleaver then by using equation interleaver. The matrix interleaving stage, a matrix will be filled row by row, the filled matrix will be sent column by column. While in the data will be rearranged depending on a certain equation in the equation interleaver that reallocate the symbols without omitting anyone. In the next stage (the modulation process), the data will be modulated using a m-modulation scheme and the generated symbols will be normalised to be sent to the OFDM transmitter [17, 18]. Between the transmitter and the receiver, an additive white Gaussian noise (AWGN) channel is 
used. The receiver of the HIPERLAN system comprises components that reverse the work of the transmitting side.

\section{Open Loop Transmit Diversity Technique with HIPERLAN/2}

Alamouti technique has been introduced to the HIPERLAN/2 system as shown in Fig. 2, where each user has two antennas in the transmitting side and one antenna for the receiving side. It is assumed that each used is working on a full-mode duplexing and the antennas for transmitting and receiving are closely located for each user.

The channel box details of Fig. 2 is shown in Fig. 3. In the channel modelling, there are two types of blocks which are the attenuation and gain block diagrams, where the channel between the transmit user and the receive user will use the attenuator, whereas within the same user, the channels between the transmitting antennas and the receive antenna will use the gain block. Thus, the user receive antenna, will receive two signals; the first from the other user and the second from the transmitting signal by himself in addition to the noise. As shown from Figure 4, a block gain will be used to amplify the symbols for the first user before transmitting it to the Rayleigh channel [21, 22]. At the same time, an attenuator will be used to attenuate the symbols that sent by the other user. After that, each set of symbols will have a channel which is independent from other channel.

The required channels will be combined for each user. It should be noticed within the same user, the channels is known between the transmit and the receive antennas in addition to the transmitted symbols [21, 22].

The HIPERLAN/2 system illustrated in Fig. 1 was evaluated when a modulation of 16QAM is used. Fig.4 displays the scattering of the symbols at: (a) the transmitter side, (b) inside the channel, and (c) at the receiver side. The effect of the channel on the transmitting symbols is obvious.

\section{Results}

The performance of the modified HIPERLAN/2 with Alamouti technique using two transmit antennas and one receive antenna using different cases of modulation schemes such as 4-, 16-, 32-, and 64-QAM is shown in Fig 5. It can be seen that; More SNR is required as the modulation complexity increases to keep the system performance within the same BER. In addition, the use of additional transmit antenna helps in achieving the same BER with lower SNR. Fig.6 shows the effect of the feedback accuracy on the performance of the modified HIPERLAN/2 with Alamouti technique using different modulation schemes, it can be seen that the feedback accuracy affects the performance significantly, as the performance will be improved as the feedback accuracy enhances. At the same time, a higher modulation scheme require a higher feedback accuracy. 


\section{Modified HIPERLAN/2}

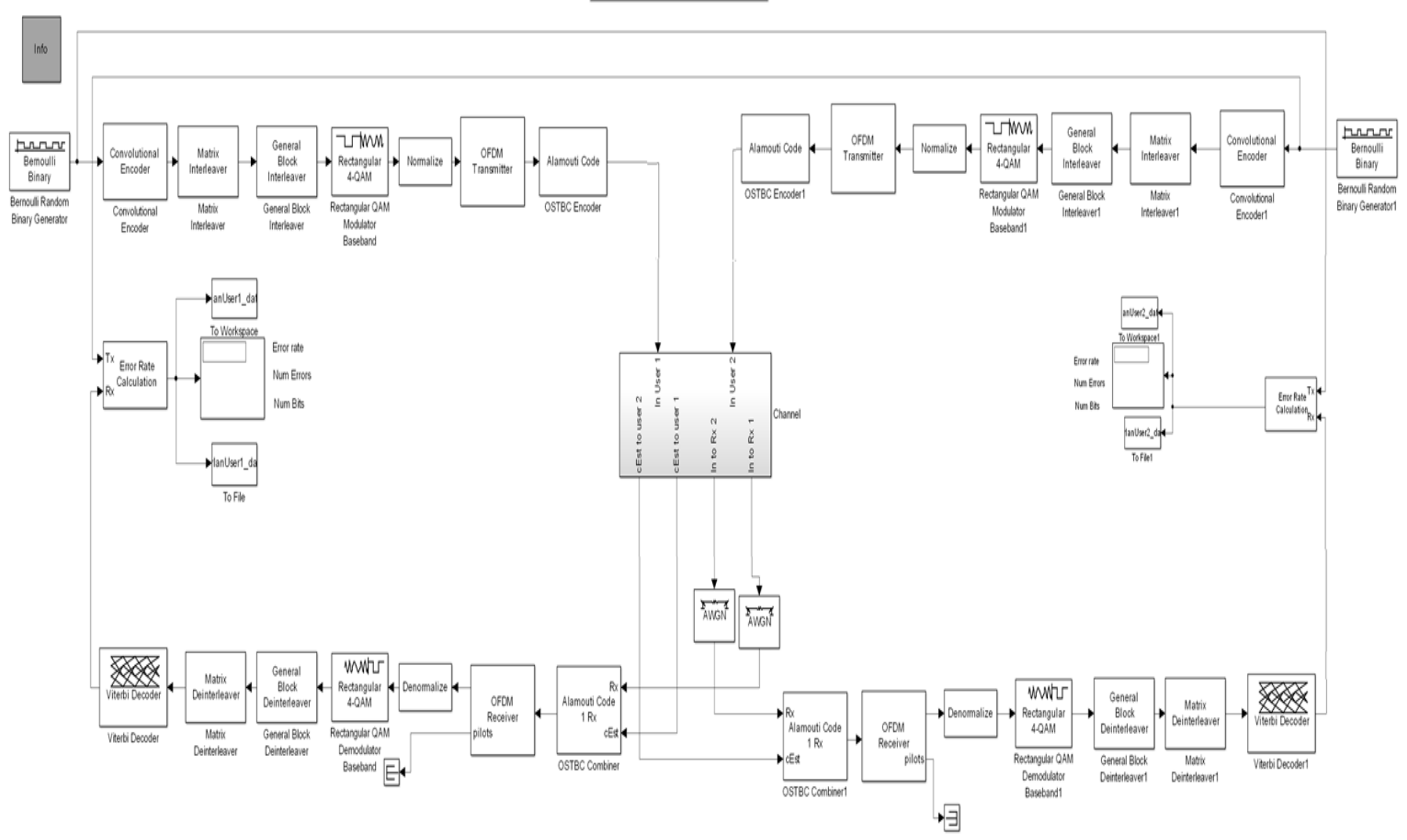

Fig. 2. Modified HIPERLAN system. 


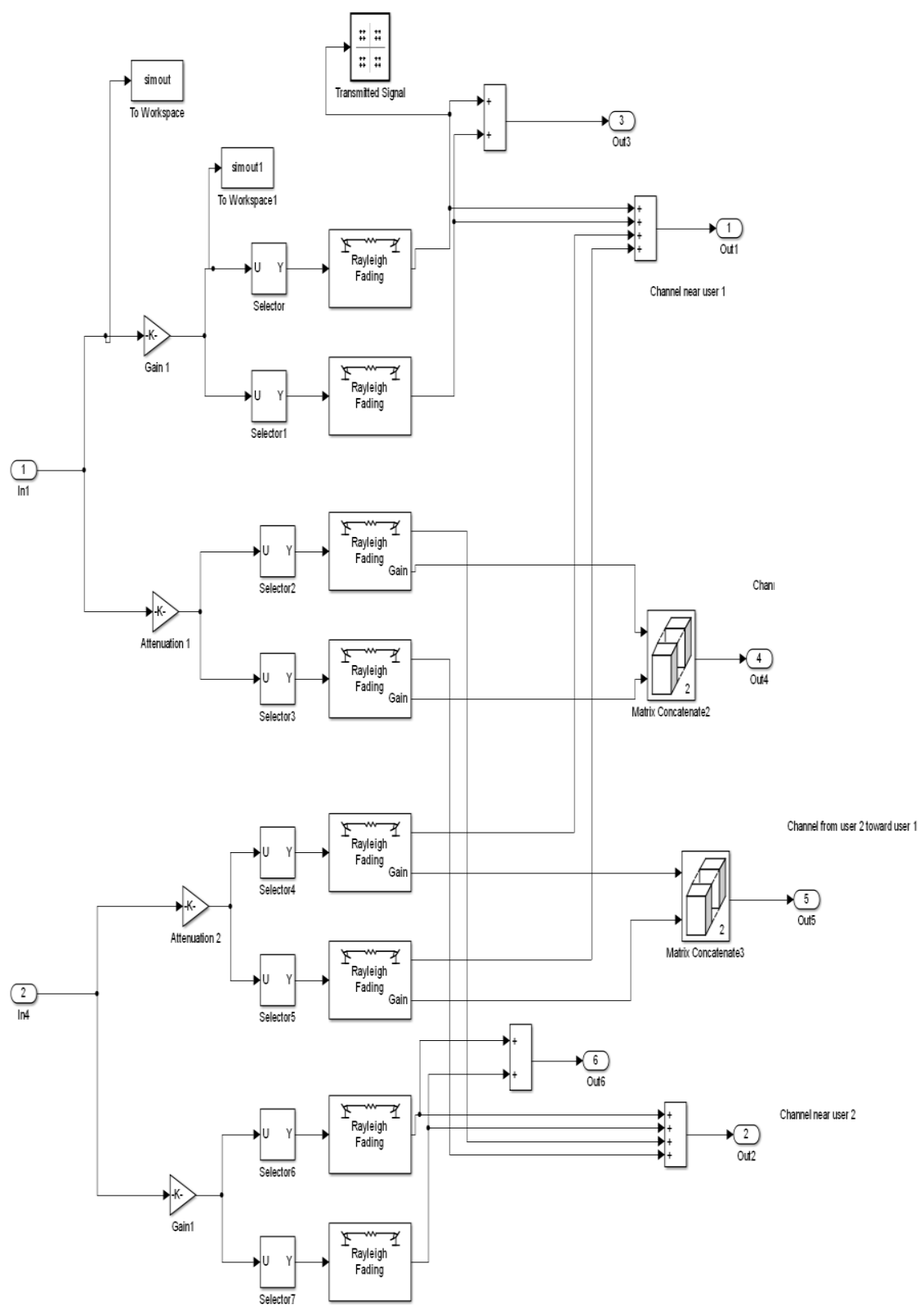

Fig.3. The channels modelling when each user has two antennas for transmit and one antenna for recieving receive. 


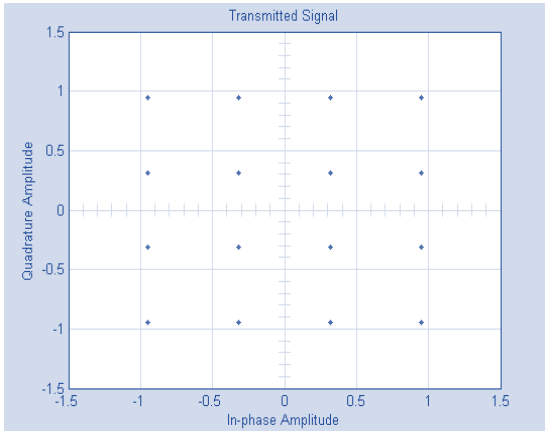

(a)

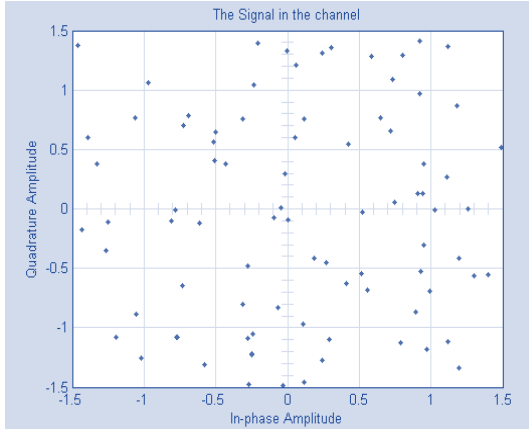

(b)

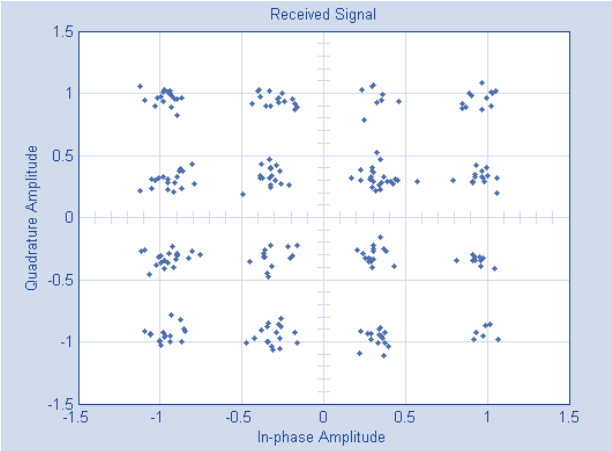

(c)

Fig. 4. The scattering of symbols at (a) the transmitting side, (b) the channel, and (c) the receiving side.

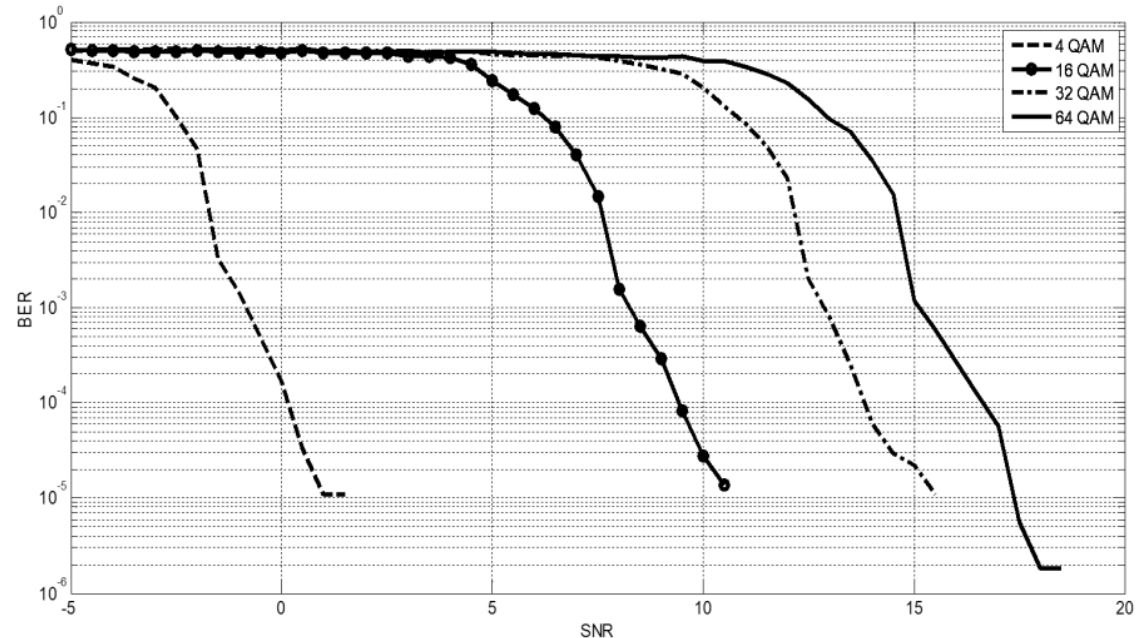

Fig 5. Modified HIPERLAN/2 system performance when Alamouti technique is applied using different modulation schemes. 



Fig6. Modified HIPERLAN/2 with Alamouti technique when feedback accuracy is varying when (a) 16-QAM (b) 32-QAM (c) 64-QAM 


\section{Conclusions}

The data rate can be increased in the HIPERLAN/2 modified system by utilizing a higher modulation scheme where the same BER can be achieved at the cost of increasing the SNR. Furthermore, introducing Alamouti technique to the HIPERLAN/2 improves system performance, such as the capacity and the data rate. A full-duplex mode is suggested, where each user can send and receive at the same time. The simulated results showed that as the modulation scheme complexity increases, more SNR is required to achieve same BER. Besides, the results illustrated that the suggested system performance is heavily depended on the feedback accuracy, where the system performance is directly proportional to the feedback accuracy.

\section{References}

[1] A. Paulraj, A. P. Rohit, R. Nabar, and D. Gore, Introduction to Space-Time Wireless Communications. Cambridge University Press, 2003.

[2] A. M. Abdulkhaleq et al., "18-W Three-Way Doherty Amplifier For 5G Applications," presented at the Antennas and Propagation Conference 2019, Birmingham, UK, 2019.

[3] H. Jafarkhani, Space-Time Coding: Theory and Practice. Cambridge University Press, 2005.

[4] A. M. Yahya et al., "Noise Cancellation for HIPERLAN/2 with Open Loop Transmit Diversity Technique," Inventions, vol. 4, no. 3, 2019, doi: 10.3390/inventions4030046.

[5] A. M. Abdulkhaleq et al., "Antennas Impedance Changing on Effect Three-Way DohertyAmplifier for Green RF Communications," presented at the EuCAP 2020, 1520-March-2020, 2020.

[6] A. M. Abdulkhaleq et al., "Recent Developments of Dual-Band Doherty Power Amplifiers for Upcoming Mobile Communications Systems," Electronics, vol. 8, no. 6, 2019, doi: 10.3390/electronics8060638.

[7] E. G. Larsson and P. Stoica, Space-Time Block Coding for Wireless Communications (Space-Time Block Coding for Wireless Communications). Cambridge University Press, 2008.

[8] Wireless World. I.P.C. Business Press Limited, 1974.

[9] A. A. Jasim, K. M. Younus, A. Ali, K. H. Sayidmarie, A. Alhaddad, and R. A. AbdAlhameed, "A simple self-interference cancellation technique for full duplex communication," in 2017 Internet Technologies and Applications (ITA), 12-15 Sept. 2017 2017, pp. 224-229, doi: 10.1109/ITECHA.2017.8101943.

[10] I. H. Nejdi, Y. Rhazi, M. A. Lafkih, and S. Bri, "Designing Multiband Multilayered Microstrip Antenna for UMTS, ISM, Communication Satellite, HiperLAN and CBand," in 2018 International Symposium on Advanced Electrical and Communication Technologies (ISAECT), 21-23 Nov. 2018 2018, pp. 1-8, doi: 10.1109/ISAECT.2018.8618728.

[11] M. S. Alam, K. M. Yeh, M. T. Islam, N. Misran, and A. M. Hasbi, "An EBG microstrip antenna for $5.4 \mathrm{GHz}$ WLAN/HIPERLAN applications," in 2012 IEEE Student Conference on Research and Development (SCOReD), 5-6 Dec. 2012 2012, pp. 144147, doi: 10.1109/SCOReD.2012.6518628.

[12] A. Gupta, M. R. Tripathy, V. Choudhary, and D. Ronnow, "A compact four-element MIMO antenna for WLAN/WiMAX and HiperLAN applications," in 2016 IEEE 
Annual India Conference (INDICON), 16-18 Dec. 2016 2016, pp. 1-4, doi: 10.1109/INDICON.2016.7838862.

[13] H. Asokan and S. Gopalakrishnan, "A Miniaturized inductive - Loaded narrow strip wide band-notched ultra-wideband monopole antenna with dual-mode resonator," AEU - International Journal of Electronics and Communications, vol. 86, pp. 125-132, 2018/03/01/ 2018, doi: https://doi.org/10.1016/j.aeue.2018.01.024.

[14] M. B. Zid, Recent Trends in Multi-user MIMO Communications. IntechOpen, 2013.

[15] A. M. Abdulkhaleq et al., "Noise cancellation for compact MIMO systems," in the 5th International Conference on Internet Technologies and Applications, 2013. [Online]. Available: http://hdl.handle.net/10454/9585

[16] WIRELESS COMMUNICATIONS. Tata McGraw-Hill Education.

[17] C. Esli, B. Ozgul, and H. Delic, "Space-frequency coded HIPERLAN/2," Consumer Electronics, IEEE Transactions on, vol. 50, no. 4, pp. 1162-1168, 2004, doi: 10.1109/TCE.2004.1362514.

[18] C. Esli, G. Ay, B. Ozgul, and H. Delic, "Space-frequency coding for the HIPERLAN/2 standard," in Electrotechnical Conference, 2004. MELECON 2004. Proceedings of the 12th IEEE Mediterranean, 12-15 May 2004 2004, vol. 2, pp. 445-448 Vol.2, doi: 10.1109/MELCON.2004.1346960.

[19] M. Moubadir, I. Badaoui, N. A. Touhami, M. Aghoutane, and M. E. Ouahabi, "A new circular polarization dual feed microstrip square patch antenna using branch coupler feeds for WLAN/HIPERLAN applications," Procedia Manufacturing, vol. 32, pp. 702-709, 2019/01/01/ 2019, doi: https://doi.org/10.1016/j.promfg.2019.02.274.

[20] J. Kruys, "HIPERLAN, applications and requirements," in Personal, Indoor and Mobile Radio Communications, 1992. Proceedings, PIMRC '92., Third IEEE International Symposium on, 19-21 Oct 1992 1992, pp. 133-138, doi: 10.1109/PIMRC.1992.279949.

[21] A. A. Giordano and A. H. Levesque, Modeling of Digital Communication Systems Using SIMULINK. Wiley, 2015.

[22] K. D. Rao, Channel Coding Techniques for Wireless Communications. Springer India, 2015. 